\title{
GUIDELINES FOR GOOD PRACTICE ON VOLTAGE QUALITY MONITORING
}

\author{
Math BOLLEN \\ LTU - Sweden \\ math.bollen@1tu.se \\ Romain CASTEL \\ CRE - France \\ romain.castel@cre.fr \\ Werner FRIEDL \\ e-control - Austria \\ werner.friedl@e-control.at \\ Ferruccio VILLA \\ AEEG - Italy \\ fvilla@autorita.energia.it
}

\author{
Philippe BAUMANN \\ Elcom - Switzerland \\ philippe.baumann@elcom.admin.ch \\ Jorge ESTEVES \\ ERSE - Portugal \\ jesteves@erse.pt \\ Samuele LARZENI \\ AEEG - Italy \\ slarzeni@autorita.energia.it \\ Lars STRÖM \\ EI - Sweden \\ lars.strom@ei.se
}

\author{
Yvonne BEYER \\ NMA - The Netherlands \\ yvonne.Beyer@nma.nl \\ Sergio FAIAS \\ ERSE - Portugal \\ SFaias@erse.pt \\ Jasmina TRHULJ \\ AERS - Serbia \\ jasmina.trhulj@aers.rs
}

\begin{abstract}
Guidelines are given for setting up and running a voltage quality monitoring programme. These guidelines are published jointly by CEER and ECRB and contain among others recommendations on the number and location of monitors, on disturbances to be monitored and indices to be calculated, on reporting of the results and on financing of the programme. It is concluded that voltage quality monitoring programs are important tools for voltage quality regulation and that all other possible applications should be kept in mind when setting up such a programme. It is also concluded that such programmes should be funded through the network tariffs; that the results should be made available regularly and that diversification of indices and methods is to be avoided.
\end{abstract}

\author{
ABBREVIATIONS \\ NRA: national regulatory authority \\ CEER: Council of European Energy Regulators \\ ECRB: Energy Community Regulatory Board \\ VQ: voltage quality \\ VQM: voltage quality monitoring \\ LV: low voltage \\ MV: medium voltage \\ $\mathrm{HV}$ : high voltage \\ EHV: extra high voltage
}

\section{INTRODUCTION}

The $5^{\text {th }}$ CEER Benchmarking report on Quality of Electricity Supply, published in 2011 [1], is a joint deliverable of the Council of European Energy Regulators (CEER) and the Energy Community Regulatory Board (ECRB). In this document, 18 countries reported to have an operational voltage quality monitoring (VQM) system. Next to that the majority of network operators in most countries perform VQM on a continuous basis at one or more locations. The number of voltage quality (VQ) monitors in operation in the public distribution and transmission networks has seen a significant growth in recent years, largely due to a number of technical developments. The cost of monitoring equipment has dropped significantly, making it no more an unsurpassable barrier to install a larger number of instruments. Also, the costs of ancillary technologies, like communication, data storage, data processing and visualisation of results have significantly decreased, whereas at the same time their performance has improved a lot. The appearance of smart meters with VQM functionality is the most visible proof of such development.

Recent and on-going developments within the power grid and equipment connected to it have further resulted in an increased interest in VQ, e.g.: photo-voltaic installations; wind power; energy-efficient lighting; HVDC; and voltage quality regulation. These developments were the driving forces behind a joined report by CEER and ECRB containing guidelines on VQM supported by both organisations [2]. This paper summarizes those guidelines; the reader is referred to the full report for more details.

\section{APPLICATIONS OF VQM}

A number of possible applications of the results obtained from VQM programmes have been identified in the report:

$\checkmark$ Compliance monitoring

$\checkmark \quad$ System performance monitoring

$\checkmark$ Specific site monitoring (complaints, pre- 


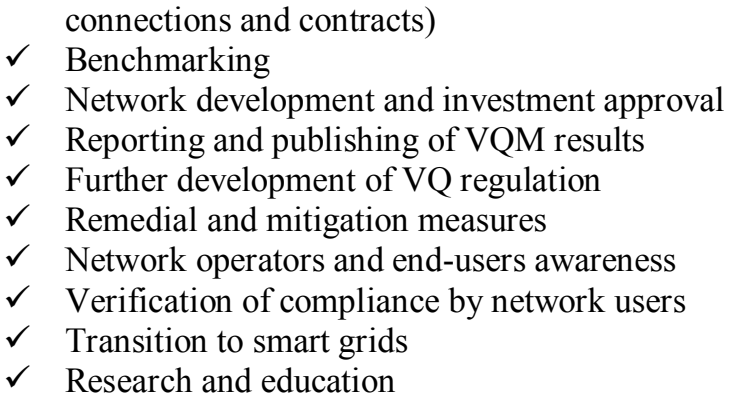

\section{VQM PROGRAMMES}

According to the possible applications listed above, it is advisable to start a VQM programme as a tool for:

$\checkmark$ continuous monitoring aimed at verification of compliance and introduction or further development of VQ regulation;

$\checkmark$ further understanding of relations between network properties, disturbances and equipment behaviour with the aim of improving compatibility between equipment and the grid;

$\checkmark$ benchmarking analysis of VQ both at national and international level.

$\checkmark$ collecting data in order to set or improve technical standardisation;

$\checkmark$ research and education, aimed at gaining knowledge from data collected, leading further to continuous VQM programmes implementation;

When a VQM programme is in place, other applications are possible in parallel, such as obtaining information on local VQ to existing users, following complaints, providing information to future users, prior to connection to the network (especially industrial users who are generally the most sensitive to VQ) or in the case of VQ contracts.

It is especially recommended that the results from VQM programmes are used for identifying and dealing with new challenges in the system, such as impact of distributed generation and new types of customers or in facilitating the transition to smarter grids.

When starting the process of setting up a VQM programme, all possibly needed applications should be considered. An initially well-designed VQM programme will allow other applications immediately or following minor inexpensive adjustments. In order to have a wider insight into required applications of VQM programmes, close cooperation between interested parties, especially NRAs, network operators and network users, but also equipment manufacturers and researchers, is recommended in the early phases of establishing a VQM programme.

\section{NUMBER AND LOCATION OF MONITORS}

The report also gives guidelines for the number of monitors, which locations in the network they should be placed and the length of the measurement period.

In EHV and HV networks, it is considered good practice to monitor the $\mathrm{VQ}$ at all $\mathrm{EHV} / \mathrm{HV}, \mathrm{EHV} / \mathrm{MV}$ and $\mathrm{HV} / \mathrm{MV}$ substations and at the connection points of all EHV and HV customers, producers (power stations) as well as consumers (industrial customers). Given that the number of monitoring points will be relatively small, the costs of continuous VQM at these points in the network are expected to be limited.

In MV networks, it is recommended to monitor the VQ at the MV busses of all EHV/MV and HV/MV transformers and at a selection of $\mathrm{MV} / \mathrm{LV}$ substations and connection points of MV customers. The exact number of measurement locations is expected to vary between countries due to differences in network structure.

In LV networks, it is considered good practice to monitor the VQ at a random selection of connection points of LV customers throughout the country for a statistically relevant sample. For VQM in the LV networks, it is possible to use both fixed and portable instruments. Portable monitors are considered acceptable as long as they are combined with a number of locations with fixed monitors. Fixed instruments will give a better overall view of the VQ at each monitoring location, but monitoring with portable instruments tends to be cheaper in capital costs and allows the monitoring of more locations. Furthermore, smart meters might become part of VQM in the future. However, smart meters are currently only able to measure a limited set of VQ disturbances.

\section{DISTURBANCES AND INDICES}

When setting up a VQM programme it is considered good practice to monitor all disturbances as listed in the European voltage-characteristic standard EN 50160. The lack of standardised measurement methods for some disturbances makes benchmarking between network operators, between regions within a country, and between countries impossible, but it does not prevent feedback to network operators, network users and NRAs on the performance of the network. Therefore it is recommended to follow the standards whenever possible and use a broad set of characteristics and indices, beyond what is used for reporting or benchmarking. There is no need to be limited to standard methods, but standard methods should be included. For benchmarking purposes, commonly-agreed indices should be used.

For the evaluation of the VQ, it is considered good practice that the following voltages are used: 
$\checkmark \quad$ For measurements in solidly-grounded LV networks (which covers the majority of LV networks in Europe) the phase-to-neutral voltage should be used for the evaluation of the VQ;

$\checkmark \quad$ In all other cases, the phase-to-phase voltages should be used.

The following is considered to be good practice where it concerns the processing of flagged values:

$\checkmark$ Flagged 10-minute values (or 1-minute values in case these are used) should be removed from the statistics for flicker, voltage unbalance, harmonic voltage and interharmonic voltage.

$\checkmark$ The same holds for 10-minute or 1-minute values during which a transient or a single rapid voltage change occurs.

$\checkmark$ For supply voltage variations only flagged values due to interruptions should be removed. All other values should be included in the calculation of the indices.

It is also recommended to keep track of the time stamps of those flagged values that have been removed from the data. When a large number of values is removed the resulting indices (e.g. over one week) will have limited value and it may be decided to not report those.

Recommendations are given in the report for the calculation of indices for benchmarking. These indices include annual indices per monitor location for each of the three phase-to-phase or phase-to-neutral voltages and annual indices for a number of monitors at similar location. The following disturbances are covered by these indices:

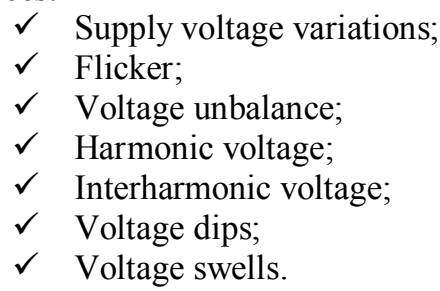

\section{REPORTING OF THE RESULTS}

Different parties are interested in the network performance concerning VQ: NRAs, network operators, individual users, research organizations. Reporting and publishing of the results from a VQM programme is thus important and it is recommended that the publishing is done in a uniform manner. Guaranteeing comparison of VQM results will push improvements in networks and will give suitable and preliminary information to network-users. This will raise the awareness of networkusers about voltage quality levels, as well as giving them suitable information for designing their installations.

NRAs should publish main results from VQM programmes, including compliance with VQ regulation and observed trends, in a report at least once a year. This report might also be used to provide information about VQ fundamentals and practical consequences of insufficient VQ. A centralised approach should be used in designing data collection and reporting system.

Data should be made available to all interested parties, when necessary according to security mechanisms aiming at protecting the interests of network operators and of individual network users. Initiatives should be taken in order to inform network users about their responsibilities.

The use of the internet is strongly encouraged for the publication of VQ data. Comparative publications are recommended for pushing improvements in networks and giving suitable and preliminary information to end-users. The use of proprietary software should be avoided in order to facilitate interoperability and promote standardised common data formats.

\section{FINANCING OF VQM}

It has become clear from the applications of VQM presented above that there are several benefits for different stakeholders of implementing a national VQM programme. However due to the costs of VQM programmes financing frameworks must be considered in order to ensure its effective implementation. The VQM financing framework should include two main steps: the costs assessment and the financing plan. VQM programme costs assessment should be performed throughout an inventory of the whole costs associated to the programme implementation (capital expenses) and maintenance (operational expenses). The most appropriate way of funding such a programme is through the grid tariffs, mostly with the contribution of all connected customers (socialised costs). However this can vary between countries based on the local tariff structure and regulation.

According to the questionnaire results, for most NRAs with national VQM programmes underway, the expenses of their programmes are not completely available. In this regard, it is recommended that the NRA follows the national VQM programmes in order to keep inventory of the expenses.

According to the current practice in most European countries with national VQM programmes underway, it is a reasonable approach to allocate the costs of a national VQM programme to all connected customers through the use of grid tariffs, provided that those costs do not exceed $0.2 \%$ of the capital and operational expenses of the grid and most customers benefit from the implementation of such programmes.

However, in order to better balance the costs and benefits of the different type of customers and avoid some sense of unfairness of the connected customers that do not expect direct advantages from a national VQM 
programme implementation, special attention should be given to grid tariffs design

The experiences reported in this GGP demonstrated that in most European countries the NRA is responsible for approving the annual budget for the national VQM programmes. However, the figures of such investments should be more detailed and transparent.

It is further recommended that network operators, where needed in cooperation with research institutes and universities, develop methods to apply VQM data towards a more cost-effective planning and operation of the electricity network. The results from this development should be disseminated at least at a European level.

\section{CONCLUSIONS}

Voltage quality monitoring programmes are important tools for voltage quality regulation

Voltage quality (VQ) is an important aspect of the service network operators provide to the network users. As such, network operators should be transparent about the level of quality they deliver. Voltage quality monitoring (VQM) programmes can facilitate the delivery of such transparency. There are sufficient applications of the data obtained from VQM and advantages to network users, to justify a VQM programme. The costs of such a programme, along the proposed lines, are a small part of the total costs of operating the electricity networks. A VQM programme can be fully run by network operators, or be installed by the NRA and operated by the network operator with NRA access to the data. Which option is most appropriate depends on national circumstances.

\section{All possible applications should be kept in mind}

When setting up a VQM programme it is important to consider all possible applications. Even if the purpose of a programme is initially limited, small changes in the setup of a programme or of parameters recorded or calculated, can allow future applications at no or very small extra cost. The setting up of such a programme should be done in close cooperation between the NRA and the other stakeholders, especially the network operators.

\section{Voltage quality monitoring programmes should be funded through the network tariffs}

It is deemed that the benefits, with regards to the wide range of possible applications, outweigh the costs ofVQM programmes. The most appropriate way of funding such a programme is through network tariffs. However this can vary between countries based on the local tariff structure and regulation.

\section{Results should be made available regularly}

Publication of the results and making data available in other ways are important parts of a VQM programme. It is recommended that the NRA publishes the main results from the programme, including compliance with voltage quality regulation and important trends, in a report at least once a year. Such a report can be published by an NRA, network operators or transmission system operators and combined with a similar report on continuity of supply and/or commercial quality.

In addition, data should be made available to other stakeholders, including the general public. Where no objections from individual network users or other important objections exist, all data should be made available for free or at a reasonable cost, for research and education purposes.

\section{Diversification of indices and methods is to be} avoided

A number of VQM programmes have already been launched in some European countries. There are large differences between these programmes making it difficult to compare the results. Such a diversification also makes it more difficult to exchange knowledge and experience. It is strongly recommended that VQM programmes are harmonised according to the guidelines proposed in the report. The need for harmonization applies to, among others, the choice of monitoring locations, types of disturbances monitored, characteristics recorded and indices calculated. Beyond the list of indices for benchmarking, which is recommended as a harmonised set of indices to be obtained from every programme where possible, each European country is recommended to obtain additional indices that specifically reflect the local circumstances.

\section{ACKNOWLEDGEMENTS}

The contributions are acknowledged from many others to the material that formed the basis for this paper, especially the other members of the Electricity Quality of Supply and Smart Grids Task Force within CEER and the Customer Working Group within the Energy Community. The text of this paper is based on the text in the Guidelines of good practice on the implementation and use of voltage quality monitoring systems for regulatory purposes (GGP). Where the text of this paper deviates from the text of the GGP, it is the personal opinion of the authors and not necessarily the opinion of CEER or ECRB, neither of the national regulatory authorities the authors work for.

\section{REFERENCES}

[1] Benchmarking Report on Quality of Electricity Supply, CEER and ECRB, December 2011.

[2] Guidelines of good practice on the implementation and use of voltage quality monitoring systems for regulatory purposes, CEER and ECRB, December 2012. 